\title{
Diversidade de formigas epigéicas (Hymenoptera, Formicidae) em três ambientes no Parque Estadual Mata dos Godoy, Londrina, Paraná
}

\author{
Danielle T. Lopes ${ }^{1}$, José Lopes ${ }^{2}$, Ivan Cardoso do Nascimento ${ }^{3} \&$ Jacques H. Delabie $^{4}$
}

1. Servidão Reduzino Vergilino Teixeira, 71, Chalé 04, Barra da Lagoa, 88061-378 Florianópolis, SC, Brasil. (danielle_thais@yahoo.com.br)

2. Departamento de Biologia Animal e Vegetal, Universidade Estadual de Londrina, Campus Universitário, 86.051-990 Londrina, PR, Brasil. (jea@uel.br)

3. Universidade Estadual do Sudoeste da Bahia, Campus de Jequié, Rua José Moreira Sobrinho, Jequiezinho, 45.206-510 Jequié, BA, Brasil. (icardoso@ hotmail.com)

4. Laboratório de Mirmecologia, Convênio CEPLAC/UESC, Caixa Postal 7, 45.600-000 Itabuna, BA, Brasil. (jacques.delabie@gmail.com)

\begin{abstract}
Epigeic ants diversity (Hymenoptera, Formicidae) in three environments in Mata dos Godoy State Park, Londrina, State of Paraná, Brazil. Considering the poor knowledge about the ant fauna of the state of Paraná, Brazil, this study aimed to compare the ant assemblages in three environments (primary forest, reforested area and secondary growth forest) of Mata dos Godoy State Park, municipality of Londrina. This study was carried out between December 2004 and March 2005. Ant collections were made using sardine baits and pitfall traps. We collected 102 ant species belonging to 38 genera of nine subfamilies. Myrmicinae was the richest subfamily (58 spp.) followed by Formicinae (20 spp.), Ponerinae (9 spp.), Ectatomminae, Heteroponerinae, Ecitoninae and Pseudomyrmecinae (3 species each), Dolichoderinae (2 spp.) and Proceratiinae (1 specie). The richest genera were Pheidole Westwood, 1839 and Camponotus Mayr, 1861, respectively with 14 and 11 species. The primary forest showed the highest richness values, number of exclusive species and diversity (92 spp., $20 \mathrm{spp}$. and $\mathrm{H}^{\prime}=3.51$, respectively), followed by the reforestation area (73 spp., $6 \mathrm{spp}$. and $\left.\mathrm{H}^{\prime}=3.47\right)$ and the secondary growth forest $\left(67 \mathrm{spp} ., 4 \mathrm{spp}\right.$. and $\left.\mathrm{H}^{\prime}=3.34\right)$. The similarity values between the three environments were rather high. In each sample series, the observed richness was between 33 and 53 species and the estimated richness was between 35 and 86 species. The occurrence of seven guilds of ants was defined: omnivore species, specialist predators, generalist predator litter, armyants, vegetation dominant, soil dominant and fungus growers.
\end{abstract}

KEYWORDS. Myrmecofauna, richness, guilds.

RESUMO. Considerando o escasso conhecimento sobre a mirmecofauna do estado do Paraná, o presente estudo objetivou comparar as assembleias de formigas encontradas em três ambientes (mata primária, área de reflorestamento e capoeira) do Parque Estadual Mata dos Godoy, Londrina, Paraná. Para as coletas foram utilizadas iscas de sardinha e armadilhas pitfalls. Foram coletadas 102 espécies, pertencentes a 38 gêneros de nove subfamílias de formigas. Myrmicinae foi a subfamília com o maior número de espécies (58 spp.), seguida por Formicinae (20 spp.), Ponerinae (9 spp.), Ectatomminae, Heteroponerinae, Ecitoninae e Pseudomyrmecinae (3 espécies cada), Dolichoderinae (2 spp.) e Proceratiinae (1 espécie). Os gêneros mais ricos em espécies foram Pheidole Westwood, 1839 e Camponotus Mayr, 1861, respectivamente com 14 e 11 espécies. A mata primária apresentou os maiores valores de riqueza, número de espécies exclusivas e diversidade (92 spp., 20 spp. e H' = 3,51, respectivamente), seguida da área de reflorestamento (73 spp., 6 spp. e H' = 3,47) e capoeira (67 spp., 4 spp. e $\left.\mathrm{H}^{\prime}=3,34\right)$. Os valores de similaridade entre os três ambientes foram próximos. A riqueza observada, em cada série de amostra, foi entre 33 e 53 espécies e a riqueza estimada foi entre 35 e 86 espécies. A ocorrência de sete guildas de formigas foi definida em espécies onívoras, predadoras especialistas, predadoras generalistas de serapilheira, formigas legionárias, arborícolas dominantes, dominantes de solo e cultivadoras de fungo.

PALAVRAS-CHAVE. Mirmecofauna, riqueza, guildas.

O conhecimento sobre comunidades de formigas é importante por fornecer uma imagem sobre a situação transitória ou permanente do ambiente avaliado, dando subsídios para inferir sobre a conservação ou degradação dos mesmos. Associado a isto, está o uso da terra pelo homem (Noss, 1990), impactos de práticas florestais e sucesso de recuperação ecológica (MAJER \& КосK, 1992), comparação de diferentes ferramentas de manejo, impacto de perturbações em áreas de conservação (Delabie et $a l .$, 2006) e avaliação da diversidade biológica (OLIVEIRA \& BRANDÃO, 1991).

Para permitir a identificação de padrões da estrutura das comunidades, independentemente de padrões biogeográficos, as formigas podem ser divididas em guildas baseadas não apenas em uma espécie ou a fauna inteira, mas em um grupo de espécies com valências ecológicas similares (ANDERSEN, 1997). Trabalhos que relacionam a distribuição de espécies de formigas com gradientes sucessionais ou diferentes práticas de uso do solo têm mostrado que a riqueza e a diversidade de espécies aumentam em ambientes mais complexos, pelo fato destes possuírem maior disponibilidade de recursos alimentares, maior especialização dos sítios de nidificação e por reduzirem a dominância de algumas espécies (MAJER \& CAMER-Pesci, 1991; Roth et al., 1994; Bustos \& UlloACHACón, 1997). Em outros estudos, pelo contrário, o aumento da complexidade estrutural do ambiente mostrou diminuição da diversidade de espécies (WISDOM \& Whitford, 1981; Torres, 1984; PuntTila et al., 1991; Lassau \& Hochuli, 2004) ou não mostrou efeito significativo sobre as comunidades de formigas (BELSHAW \& Bolton, 1993). Isso sugere a necessidade de mais estudos em escalas locais que proporcionem informações sobre as relações entre as espécies de formigas e os ecossistemas naturais ou modificados, na busca de um padrão para este tipo de enfoque. 
Considerando o pouco conhecimento da mirmecofauna do Paraná, este estudo objetivou comparar a riqueza e a diversidade da mirmecofauna em ambientes de mata primária, capoeira e reflorestamento no Parque Estadual Mata dos Godoy e classificar a mirmecofauna em guildas.

\section{MATERIAL E MÉTODOS}

O Parque Estadual Mata dos Godoy localiza-se no município de Londrina no norte do estado do Paraná, e seu centro geográfico situa-se a $23^{\circ} 27^{\prime} \mathrm{S}$ e $51^{\circ} 15^{\prime} \mathrm{W}$. O parque é uma das últimas reservas naturais de mata nativa desta região, possuindo 680 ha, sendo que destes, 570 ha são constituídos por floresta contínua e os 110 ha restantes constituem áreas de reflorestamento e de floresta secundária (capoeiras). O estudo foi desenvolvido nestes três diferentes ambientes. A capoeira, com cerca de 20 anos de idade apresenta uma área de aproximadamente 30 ha no lado sudeste do parque. A área de reflorestamento encontra-se no lado leste do parque, com idade de 15 anos e apresenta uma área de 40 ha. A área está reflorestada com Colubrina glandulosa (Rhamnaceae), Cordia trichotoma (Boraginaceae), Parapiptadenia rigida (Leguminosae, Mimosoideae), Pelthophorum dubium (Leguminosae, Caesalpinoideae) e Tabebuia impetiginosa (Bignoniaceae), com espaçamento de $3 \mathrm{~m}$ x $3 \mathrm{~m}$. O sub-bosque é dominado pelo capim-colonião, Panicum maximum (Poaceae) (M. Silveira, com. pess.).

O experimento foi realizado no período de dezembro de 2004 a março de 2005. As coletas foram feitas com armadilhas do tipo pitfall e iscas de sardinha (detalhes dessas metodologias para estudo de comunidade de formigas, ver Bestelmeyer et al., 2000). Para as amostragens com pitfall, no ambiente de mata primária, distando $200 \mathrm{~m}$ da borda, foram determinados nove locais de coletas, equidistantes $200 \mathrm{~m}$ (Fig. 1). Em cada local foram traçados dois transectos de $100 \mathrm{~m}$ de comprimento, dispostos a intervalos de $10 \mathrm{~m}$ entre si, totalizando 18 transectos com 10 armadilhas em cada um deles (180 pitfalls). Para a capoeira e o reflorestamento, seguiu-se o mesmo procedimento de distribuição das armadilhas da mata primária, porém foram determinados somente três locais de coletas para cada um destes ambientes, já que

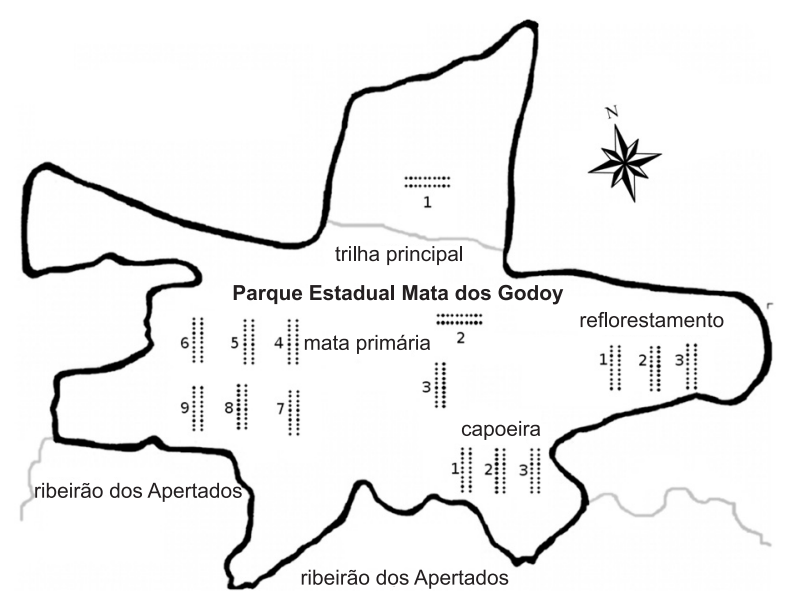

Figura 1. Localização dos pontos de coletas e armadilhas dentro do Parque Estadual Mata dos Godoy, Londrina, PR. os mesmos apresentavam tamanhos reduzidos em relação à mata, totalizando 120 pitfalls (Fig. 1). A determinação e distribuição de nove sítios de estudos no fragmento florestal visou obtenção de amostras nas diferentes condições topográficas, apresentando um platô na região norte com altitude entre $610 \mathrm{~m}$ a $650 \mathrm{~m}$, seguindo em direção à região sul, que apresenta um declive moderado, com afloramento de rocha basáltica, até atingir uma área de inundação, às margens do ribeirão dos Apertados, com uma altitude entre $460 \mathrm{~m}$ a $480 \mathrm{~m}$ (Fig. 1).

Os três ambientes juntos somam 30 transectos e 300 pitfalls. Os pitfalls consistiram em copos plásticos de $200 \mathrm{ml}$, com $70 \mathrm{ml}$ de água e cinco gotas de detergente. O tempo de exposição das armadilhas foi de 48 horas.

As iscas de atração foram compostas de sardinha conservada em óleo vegetal $\left(1 \mathrm{~cm}^{3}\right)$. As iscas foram dispostas a aproximadamente $2 \mathrm{~m}$ dos pitfalls, totalizando 300 iscas nos três ambientes. Elas ficaram expostas no campo por 90 minutos. As coletas foram realizadas sempre no período diurno, entre 09:00 h e 12:00 h.

Os Formicidae estão depositados na coleção de insetos do Laboratório de Entomologia da Universidade Estadual de Londrina, PR e no Laboratório de Mirmecologia do CEPEC/CEPLAC, Ilhéus, BA.

As espécies foram identificadas até gênero ou espécie, seguindo Bolton et al. (2006). Foram determinados os índices de diversidade de ShannonWiener ( $\left.\mathrm{H}^{\prime}\right)$, de similaridade de Jaccard $(\mathrm{J})$ e de riqueza estimada Chao2 (Colwell, 2005), uma curva de riqueza acumulada foi desenhada para cada série de transectos em cada ambiente e calculada a frequência absoluta de cada espécie. Esses índices foram determinados com o auxílio do Programa EstimateS (Statistical Estimation of Species Richness and Shared Species from Samples) versão 7.5 (Colwell, 2005). A classificação dos gêneros por guildas foi adaptada a partir do modelo proposto por Delabie et al. (2000).

\section{RESULTADOS E DISCUSSÃO}

Foram coletadas 102 espécies, pertencentes a 38 gêneros de formigas (Tab. I). Das 21 subfamílias de formigas reconhecidas por BoLTON (2003), 14 ocorrem no Brasil e, neste estudo, nove foram representadas. Myrmicinae foi a subfamília com o maior número de gêneros (22) e espécies (58 spp.) seguida por Formicinae (20 spp.), Ponerinae (9 spp.), Ectatomminae (3 spp.), Heteroponerinae (3 spp.), Ecitoninae (3 spp.), Pseudomyrmecinae (3 spp.), Dolichoderinae (2 spp.) e Proceratiinae (1 espécie) (Tab. I). Estes dados corroboram diversos estudos (SILVA \& LOPES, 1997; BRUHL et al., 1998; Silva \& SiLVESTRE, 2000, 2004) que apontam a subfamília Myrmicinae como a mais frequente e abundante observada na serapilheira da região Neotropical.

Pheidole Westwood, 1839 foi o gênero com a maior riqueza específica e um dos mais frequentes nas amostras. A dominância desse gênero ocorre pelo seu eficiente recrutamento, o que lhe permite dominar os recursos alimentares e excluir de maneira eficiente seus competidores (FowLER, 1993). É um gênero hiperdiverso (WILson, 2003), considerado não especialista, com espécies de ecologia muito diversificada e algumas altamente competitivas (HöLLDOBLER \& WILSON, 1990). A alta frequência de Pheidole sp. do grupo fallax nos locais 
Tabela I. Ocorrência de espécies de formigas epigéicas, ordenadas por subfamília, em três ambientes do Parque Estadual Mata dos Godoy, Londrina, PR, entre dezembro de 2004 e março de 2005 (M, mata; C, capoeira; R, área de reflorestamento; P, pitfall; I, isca; Fq (\%), frequência relativa de ocorrência).

\begin{tabular}{|c|c|c|c|c|c|c|}
\hline Táxons & M & $\mathrm{C}$ & $\mathrm{R}$ & $\mathrm{P}$ & $\mathrm{I}$ & $\mathrm{Fq}(\%)$ \\
\hline \multicolumn{7}{|l|}{ Subfamília Myrmicinae } \\
\hline Acromyrmex aspersus (F. Smith, 1858) & $\mathrm{X}$ & & & $\mathrm{X}$ & & 1,3 \\
\hline A. coronatus (Fabricius, 1804) & $\mathrm{X}$ & & & $\mathrm{X}$ & & 0,6 \\
\hline A. fracticornis Forel, 1909 & $\mathrm{X}$ & & & $\mathrm{X}$ & $\mathrm{X}$ & 0,6 \\
\hline A. rugosus (F. Smith, 1858) & $\mathrm{X}$ & & & $\mathrm{X}$ & & 1,3 \\
\hline Acromyrmex sp. 1 & $\mathrm{X}$ & $\mathrm{X}$ & $\mathrm{X}$ & $\mathrm{X}$ & & 1,6 \\
\hline Acromyrmex sp. 2 & $\mathrm{X}$ & & $\mathrm{X}$ & $\mathrm{X}$ & & 1,0 \\
\hline Apterostigma sp. 1 & $\mathrm{X}$ & & & $\mathrm{X}$ & & 1,3 \\
\hline Apterostigma sp. 2 & $\mathrm{X}$ & $\mathrm{X}$ & $\mathrm{X}$ & $\mathrm{X}$ & & 2,0 \\
\hline Apterostigma sp. 3 & $\mathrm{X}$ & & & $\mathrm{X}$ & & 0,6 \\
\hline Cephalotes grandinosus (F. Smith, 1860) & $\mathrm{X}$ & & & & & 0,3 \\
\hline C. pusillus (Klug, 1824) & & & $\mathrm{X}$ & $\mathrm{X}$ & $\mathrm{X}$ & 0,3 \\
\hline Crematogaster acuta (Fabricius, 1804) & $\mathrm{X}$ & $\mathrm{X}$ & $\mathrm{X}$ & $\mathrm{X}$ & $\mathrm{X}$ & 5,3 \\
\hline Crematogaster sp. 1 & $\mathrm{X}$ & $\mathrm{X}$ & $\mathrm{X}$ & $\mathrm{X}$ & $\mathrm{X}$ & 3,3 \\
\hline Crematogaster sp. 2 & $\mathrm{X}$ & $\mathrm{X}$ & $\mathrm{X}$ & $\mathrm{X}$ & $\mathrm{X}$ & 1,6 \\
\hline Cyphomyrmex bicarinatus Snelling \& Longino, 1992 & $\mathrm{X}$ & $\mathrm{X}$ & $\mathrm{X}$ & $\mathrm{X}$ & & 3,3 \\
\hline C. major Forel, 1901 & $\mathrm{X}$ & $\mathrm{X}$ & $\mathrm{X}$ & $\mathrm{X}$ & & 5,0 \\
\hline C. salvini Forel, 1899 & $\mathrm{X}$ & $\mathrm{X}$ & $\mathrm{X}$ & $\mathrm{X}$ & & 6,0 \\
\hline Cyphomyrmex sp. & $\mathrm{X}$ & $\mathrm{X}$ & $\mathrm{X}$ & $\mathrm{X}$ & & 4,6 \\
\hline Eurhopalothrix bruchi (Santschi, 1922) & $\mathrm{X}$ & & & $\mathrm{X}$ & & 0,3 \\
\hline Hylomyrma reitteri (Mayr, 1887) & $\mathrm{X}$ & $\mathrm{X}$ & $\mathrm{X}$ & $\mathrm{X}$ & & 4,3 \\
\hline Megalomyrmex drifti Kempf, 1961 & $\mathrm{X}$ & & & $\mathrm{X}$ & $\mathrm{X}$ & 0,3 \\
\hline Monomorium pharaonis (L., 1758) & $\mathrm{X}$ & $\mathrm{X}$ & $\mathrm{X}$ & $\mathrm{X}$ & & 12,0 \\
\hline Mycocepurus smithii (Forel, 1893) & $\mathrm{X}$ & $\mathrm{X}$ & $\mathrm{X}$ & $\mathrm{X}$ & $\mathrm{X}$ & 6,0 \\
\hline Myrmicocrypta sp. & & $\mathrm{X}$ & & $\mathrm{X}$ & & 0,6 \\
\hline Nesomyrmex asper (Mayr, 1887) & & $\mathrm{X}$ & & $\mathrm{X}$ & & 0,3 \\
\hline Octostruma balzani (Emery, 1894) & $\mathrm{X}$ & & & $\mathrm{X}$ & & 0,6 \\
\hline O. rugifera Mayr, 1887 & $\mathrm{X}$ & $\mathrm{X}$ & & $\mathrm{X}$ & & 1,3 \\
\hline Oxуероесиs sp. & $\mathrm{X}$ & $\mathrm{X}$ & $\mathrm{X}$ & $\mathrm{X}$ & $\mathrm{X}$ & 16,3 \\
\hline Pheidole fimbriata Roger, 1863 & $\mathrm{X}$ & $\mathrm{X}$ & $\mathrm{X}$ & $\mathrm{X}$ & $\mathrm{X}$ & 4,0 \\
\hline P. gertrudae Forel, 1886 & $\mathrm{X}$ & $\mathrm{X}$ & & $\mathrm{X}$ & $\mathrm{X}$ & 8,3 \\
\hline P. jelskii Mayr, 1884 & $\mathrm{X}$ & $\mathrm{X}$ & $\mathrm{X}$ & $\mathrm{X}$ & $\mathrm{X}$ & 28,0 \\
\hline P. radoszkowskii Mayr, 1884 & $\mathrm{X}$ & $\mathrm{X}$ & $\mathrm{X}$ & $\mathrm{X}$ & $\mathrm{X}$ & 12,6 \\
\hline Pheidole sp. 1 grupo diligens & $\mathrm{X}$ & $\mathrm{X}$ & $\mathrm{X}$ & $\mathrm{X}$ & $\mathrm{X}$ & 35,3 \\
\hline Pheidole sp. 2 grupo fallax & $\mathrm{X}$ & $\mathrm{X}$ & $\mathrm{X}$ & $\mathrm{X}$ & $\mathrm{X}$ & 53,0 \\
\hline Pheidole sp. 3 grupo tristis & $\mathrm{X}$ & $\mathrm{X}$ & $\mathrm{X}$ & $\mathrm{X}$ & $\mathrm{X}$ & 7,6 \\
\hline Pheidole sp. 4 grupo tristis & $\mathrm{X}$ & $\mathrm{X}$ & $\mathrm{X}$ & $\mathrm{X}$ & $\mathrm{X}$ & 24,3 \\
\hline Pheidole sp. 5 grupo fallax & $\mathrm{X}$ & $\mathrm{X}$ & $\mathrm{X}$ & $\mathrm{X}$ & $\mathrm{X}$ & 14,3 \\
\hline Pheidole sp. 6 grupo tristis & $\mathrm{X}$ & $\mathrm{X}$ & $\mathrm{X}$ & $\mathrm{X}$ & $\mathrm{X}$ & 38,3 \\
\hline Pheidole sp. 7 grupo flavens & $\mathrm{X}$ & $\mathrm{X}$ & $\mathrm{X}$ & $\mathrm{X}$ & $\mathrm{X}$ & 13,3 \\
\hline Pheidole sp. 8 grupo tristis & $\mathrm{X}$ & $\mathrm{X}$ & $\mathrm{X}$ & $\mathrm{X}$ & $\mathrm{X}$ & 29,6 \\
\hline Pheidole sp. 9 grupo flavens & $\mathrm{X}$ & $\mathrm{X}$ & $\mathrm{X}$ & $\mathrm{X}$ & $\mathrm{X}$ & 16,3 \\
\hline Pheidole sp. 10 grupo tristis & $\mathrm{X}$ & $\mathrm{X}$ & $\mathrm{X}$ & $\mathrm{X}$ & $\mathrm{X}$ & 23,6 \\
\hline Procryptocerus sp. & & $\mathrm{X}$ & & $\mathrm{X}$ & & 0,3 \\
\hline Pyramica appretiata (Borgmeier, 1954) & $\mathrm{X}$ & & $\mathrm{X}$ & $\mathrm{X}$ & & 4,6 \\
\hline P. eggersi (Emery, 1890) & $\mathrm{X}$ & $\mathrm{X}$ & $\mathrm{X}$ & $\mathrm{X}$ & & 6,3 \\
\hline Rogeria besuchet Kugler, 1994 & & & $\mathrm{X}$ & $\mathrm{X}$ & & 0,3 \\
\hline Solenopsis invicta Buren, 1972 & & & $\mathrm{X}$ & $\mathrm{X}$ & $\mathrm{X}$ & 0,3 \\
\hline Solenopsis sp. 1 & $\mathrm{X}$ & $\mathrm{X}$ & $\mathrm{X}$ & $\mathrm{X}$ & $\mathrm{X}$ & 61,3 \\
\hline Solenopsis sp. 2 & $\mathrm{X}$ & $\mathrm{X}$ & $\mathrm{X}$ & $\mathrm{X}$ & $\mathrm{X}$ & 40,3 \\
\hline Solenopsis sp. 3 & $\mathrm{X}$ & $\mathrm{X}$ & $\mathrm{X}$ & $\mathrm{X}$ & $\mathrm{X}$ & 16,3 \\
\hline Strumigenys elongata Roger, 1863 & $\mathrm{X}$ & & & $\mathrm{X}$ & & 3,0 \\
\hline S. infidelis Santschi, 1919 & $\mathrm{X}$ & $\mathrm{X}$ & $\mathrm{X}$ & $\mathrm{X}$ & & 3,0 \\
\hline Trachymyrmex sp. 1 & $\mathrm{X}$ & & $\mathrm{X}$ & $\mathrm{X}$ & & 2,0 \\
\hline Trachymyrmex sp. 2 & $\mathrm{X}$ & & & $\mathrm{X}$ & & 2,0 \\
\hline Trachymyrmex sp. 3 & $\mathrm{X}$ & & $\mathrm{X}$ & $\mathrm{X}$ & & 1,3 \\
\hline Wasmannia auropunctata (Roger, 1863) & $\mathrm{X}$ & $\mathrm{X}$ & $\mathrm{X}$ & $\mathrm{X}$ & $\mathrm{X}$ & 6,3 \\
\hline Wasmannia sp. 1 & $\mathrm{X}$ & $\mathrm{X}$ & $\mathrm{X}$ & $\mathrm{X}$ & $\mathrm{X}$ & 7,0 \\
\hline Wasmannia sp. 2 & $\mathrm{X}$ & $\mathrm{X}$ & $\mathrm{X}$ & $\mathrm{X}$ & $\mathrm{X}$ & 4,3 \\
\hline \multicolumn{7}{|l|}{ Subfamília Formicinae } \\
\hline Brachymyrmex heeri Forel, 1874 & $\mathrm{X}$ & $\mathrm{X}$ & $\mathrm{X}$ & $\mathrm{X}$ & $\mathrm{X}$ & 8,3 \\
\hline Brachymyrmex sp. 1 & $\mathrm{X}$ & & & $\mathrm{X}$ & & 2,0 \\
\hline Brachymyrmex sp. 2 & $\mathrm{X}$ & & & $\mathrm{X}$ & $\mathrm{X}$ & 1,6 \\
\hline Brachymyrmex sp. 3 & $\mathrm{X}$ & $\mathrm{X}$ & $\mathrm{X}$ & $\mathrm{X}$ & $\mathrm{X}$ & 3,3 \\
\hline Brachymyrmex sp. 4 & $\mathrm{X}$ & $\mathrm{X}$ & & $\mathrm{X}$ & $\mathrm{X}$ & 3,0 \\
\hline Camponotus atriceps (Smith, 1858) & $\mathrm{X}$ & $\mathrm{X}$ & $\mathrm{X}$ & $\mathrm{X}$ & $\mathrm{X}$ & 5,6 \\
\hline C. cingulatus (Mayr, 1862) & $\mathrm{X}$ & $\mathrm{X}$ & $\mathrm{X}$ & $\mathrm{X}$ & $\mathrm{X}$ & 4,3 \\
\hline C. crassus Mayr, 1862 & $\mathrm{X}$ & $\mathrm{X}$ & $\mathrm{X}$ & $\mathrm{X}$ & $\mathrm{X}$ & 5,0 \\
\hline C. lespesii Forel, 1886 & $\mathrm{X}$ & $\mathrm{X}$ & $\mathrm{X}$ & $\mathrm{X}$ & & 6,0 \\
\hline C. sericeiventris (Guérin-Meneville, 1838) & $\mathrm{X}$ & & & $\mathrm{X}$ & $\mathrm{X}$ & 0,6 \\
\hline
\end{tabular}


Tabela I (cont.)

\begin{tabular}{|c|c|c|c|c|c|c|}
\hline Táxons & $\mathrm{M}$ & $\mathrm{C}$ & $\mathrm{R}$ & $\mathrm{P}$ & $\mathrm{I}$ & $\mathrm{Fq}(\%)$ \\
\hline C. vittatus Forel, 1904 & $\mathrm{X}$ & & $\mathrm{X}$ & $\mathrm{X}$ & & 6,0 \\
\hline Camponotus sp. 1 & $\mathrm{X}$ & $\mathrm{X}$ & $\mathrm{X}$ & $X$ & $\mathrm{X}$ & 4,0 \\
\hline Camponotus sp. 2 & $\mathrm{X}$ & $\mathrm{X}$ & $\mathrm{X}$ & $X$ & & 5,0 \\
\hline Camponotus sp. 3 & $\mathrm{X}$ & $\mathrm{X}$ & $\mathrm{X}$ & $\mathrm{X}$ & $\mathrm{X}$ & 3,0 \\
\hline Camponotus sp. 4 & $\mathrm{X}$ & $\mathrm{X}$ & $\mathrm{X}$ & $\mathrm{X}$ & & 3,3 \\
\hline Camponotus sp. 5 & $\mathrm{X}$ & & $\mathrm{X}$ & $\mathrm{X}$ & $\mathrm{X}$ & 4,0 \\
\hline Myrmelachista sp. & $\mathrm{X}$ & & & $\mathrm{X}$ & $\mathrm{X}$ & 3,0 \\
\hline Paratrechina sp. 1 & $\mathrm{X}$ & $\mathrm{X}$ & $\mathrm{X}$ & $\mathrm{X}$ & $\mathrm{X}$ & 9,0 \\
\hline Paratrechina sp. 2 & $\mathrm{X}$ & $\mathrm{X}$ & & $\mathrm{X}$ & $\mathrm{X}$ & 4,6 \\
\hline Paratrechina sp. 3 & $\mathrm{X}$ & $\mathrm{X}$ & & $X$ & $\mathrm{X}$ & 2,0 \\
\hline \multicolumn{7}{|l|}{ Subfamília Ponerinae } \\
\hline Hypoponera sp. 1 & $\mathrm{X}$ & & $\mathrm{X}$ & $\mathrm{X}$ & & 2,0 \\
\hline Hypoponera sp. 2 & $\mathrm{X}$ & $\mathrm{X}$ & $\mathrm{X}$ & $\mathrm{X}$ & & 2,0 \\
\hline Hypoponera sp. 3 & $\mathrm{X}$ & & $\mathrm{X}$ & $X$ & & 0,6 \\
\hline Hypoponera sp. 4 & $\mathrm{X}$ & $\mathrm{X}$ & $\mathrm{X}$ & $\mathrm{X}$ & & 2,3 \\
\hline Hypoponera sp. 5 & $\mathrm{X}$ & $\mathrm{X}$ & $\mathrm{X}$ & $\mathrm{X}$ & & 2,0 \\
\hline Leptogenys dasygyna Wheeler, 1923 & & & $\mathrm{X}$ & $X$ & & 0,3 \\
\hline Odontomachus chelifer (Latreille, 1802) & $\mathrm{X}$ & $\mathrm{X}$ & $\mathrm{X}$ & $\mathrm{X}$ & $\mathrm{X}$ & 8,6 \\
\hline Pachycondyla harpax (Fabricius, 1804) & $\mathrm{X}$ & $\mathrm{X}$ & $\mathrm{X}$ & $\mathrm{X}$ & $\mathrm{X}$ & 8,6 \\
\hline P. striata $\mathrm{F}$. Smith, 1858 & $\mathrm{X}$ & $\mathrm{X}$ & $\mathrm{X}$ & $\mathrm{X}$ & $\mathrm{X}$ & 39,6 \\
\hline \multicolumn{7}{|l|}{ Subfamília Ectatomminae } \\
\hline Ectatomma edentatum Roger, 1863 & $\mathrm{X}$ & $\mathrm{X}$ & $\mathrm{X}$ & $\mathrm{X}$ & $\mathrm{X}$ & 25,0 \\
\hline Gnamptogenys striatula Mayr, 1884 & $\mathrm{X}$ & $\mathrm{X}$ & $\mathrm{X}$ & $X$ & $\mathrm{X}$ & 14,6 \\
\hline Gnamptogenys sp. & $\mathrm{X}$ & $\mathrm{X}$ & $\mathrm{X}$ & $X$ & $\mathrm{X}$ & 7,0 \\
\hline \multicolumn{7}{|l|}{ Subfamília Heteroponerinae } \\
\hline Heteroponera sp. 1 & $\mathrm{X}$ & $\mathrm{X}$ & $\mathrm{X}$ & $X$ & & 2,6 \\
\hline Heteroponera sp. 2 & $\mathrm{X}$ & & & $X$ & $\mathrm{X}$ & 2,6 \\
\hline Heteroponera sp. 3 & $\mathrm{X}$ & $\mathrm{X}$ & $\mathrm{X}$ & $\mathrm{X}$ & & 2,6 \\
\hline \multicolumn{7}{|l|}{ Subfamília Ecitoninae } \\
\hline Labidus coecus (Latreille, 1802) & $\mathrm{X}$ & & & $X$ & & 0,6 \\
\hline L. praedator (F. Smith, 1858) & $\mathrm{X}$ & & & $X$ & & 0,3 \\
\hline Nomamyrmex esenbeckii (Westwood, 1842) & $\mathrm{X}$ & & & $\mathrm{X}$ & & 0,6 \\
\hline \multicolumn{7}{|l|}{ Subfamília Pseudomyrmecinae } \\
\hline Pseudomyrmex gracilis (Fabricius, 1804) & & & $\mathrm{X}$ & $\mathrm{X}$ & & 0,6 \\
\hline P. rochai (Forel, 1912) & & & $\mathrm{X}$ & $\mathrm{X}$ & & 0,6 \\
\hline Pseudomyrmex sp. grupo pallidus & $\mathrm{X}$ & & $\mathrm{X}$ & $\mathrm{X}$ & & 1,0 \\
\hline \multicolumn{7}{|l|}{ Subfamília Dolichoderinae } \\
\hline Linepithema humile (Mayr, 1868) & $\mathrm{X}$ & $\mathrm{X}$ & $\mathrm{X}$ & $\mathrm{X}$ & $\mathrm{X}$ & 7,6 \\
\hline Linepithema sp. & $\mathrm{X}$ & $\mathrm{X}$ & $\mathrm{X}$ & $\mathrm{X}$ & $\mathrm{X}$ & 7,3 \\
\hline \multicolumn{7}{|l|}{ Subfamília Proceratiinae } \\
\hline Discothyrea sexarticulata Borgmeier, 1954 & & $\mathrm{X}$ & & $\mathrm{X}$ & & 0,3 \\
\hline Total de espécies: 102 & 92 & 67 & 73 & 101 & 53 & \\
\hline
\end{tabular}

estudados, pode ser explicada pelo pioneirismo de muitas espécies desse gênero que têm uma tendência a ser ubíquas em áreas perturbadas, pelo menos em numerosas regiões da América do Sul.

O gênero Camponotus Mayr, 1861 apresentou a segunda maior riqueza específica. Este gênero possui um dos maiores números de espécies descritas em nível mundial (HÖLldobler \& Wilson, 1990), tem ampla distribuição geográfica e grande diversidade de adaptações ecológicas. É constituído por espécies arborícolas e terrícolas, com dieta bastante variada, muitas possuem alta capacidade de invasão e adaptação para interagir com outros organismos (WILSON, 1976).

Em relação à frequência de captura, as espécies Solenopsis sp. 1 (61,3\%) e Pheidole sp. 2 (53\%) foram as mais comuns. Solenopsis Westwood, 1841 possui mais de 108 espécies com nomes válidos na região Neotropical (Bolton, 1995). Sabe-se que as espécies deste gênero estão entre as mais agressivas na utilização de recursos em serapilheira, principalmente as do antigo subgênero Diplorhoptrum Mayr, 1855, minúsculas e monomórficas (FERnANDEZ, 2003) sendo frequentes em ambientes agrícolas e nativos (DelabIE \& FowleR, 1995). Todas as Solenopsis coletadas no presente experimento pertencem a este grupo de espécies.
A mata primária apresentou os maiores valores de riqueza, com 92 espécies no total e 20 espécies exclusivas, e de diversidade de $\mathrm{H}^{\prime}=3,51$, seguida da área de reflorestamento com um total de 73 espécies e seis espécies exclusivas e H' = 3,47, e da capoeira, com 67 espécies no total, com quatro espécies exclusivas e $\mathrm{H}^{\prime}=3,34$.

Pode-se inferir que a maior riqueza de espécies observada na mata primária decorre da maior área e número de substratos aproveitáveis por formigas, para nidificação ou forrageio. Segundo Bustos \& UlloA-Chacón (1997), essas condições permitem a certas espécies ocupar nichos mais específicos e evitar a competição, aumentando assim a diversidade. Resultados semelhantes foram obtidos por ANDERSEN (1984), MARSH (1986), MATOS et al. (1994) e Oliveira et al. (1995), que constataram que hábitats com vegetação mais complexa permitem a coexistência de um maior número de espécies, com padrões de dominância reduzidos.

Apesar disso, percebe-se que os índices de diversidade para os três ambientes foram semelhantes. Constatou-se que, das 102 espécies encontradas, 58 foram comuns aos três ambientes. Belshaw \& Bolton (1993) não observaram diferenças quanto à composição e riqueza de espécies em áreas de floresta primária, secundária e plantios comerciais de cacau. Segundo os 
autores, esses três ambientes provavelmente oferecem micro-hábitats bastante semelhantes na serapilheira. Sendo assim, áreas remanescentes de florestas primárias são necessárias como fontes de recolonização de hábitats alterados. Os resultados obtidos corroboram essa hipótese e sugerem que a mirmecofauna dos ambientes nativos é, de certa forma, preservada na capoeira e no reflorestamento, tornando esses dois ambientes também importantes na manutenção da diversidade regional e na preservação de espécies de bosques ou de vegetação natural. Embora a capoeira e o reflorestamento tenham apresentado um número menor de espécies exclusivas do que a mata primária, deve-se levar em conta que, para propósitos de conservação, informações sobre qualidade ou vulnerabilidade biológica podem ser mais importantes do que quantidade de espécies (RAMOS-SuÁrEZ et al., 2002).

Os valores de similaridade entre os ambientes foram: mata e capoeira: $\mathrm{J}=0,656$; mata e reflorestamento: $\mathrm{J}=0,673$ e capoeira e reflorestamento: $\mathrm{J}=0,682$. Essa alta similaridade de espécies entre os ambientes pode indicar o sucesso de recuperação das áreas de capoeira e reflorestamento. A presença de muitas espécies de formigas generalistas e oportunistas nos fragmentos estudados pode também ter contribuído com a similaridade entre os ambientes.

As maiores riquezas observadas e estimadas foram encontradas na mata (53 e 86, respectivamente). Para a capoeira e o reflorestamento, esses valores foram próximos (capoeira: 37 e 44; reflorestamento: 41 e 48) (Tab. II). Analisando-se as curvas de suficiência amostral com os estimadores analíticos de riqueza de espécies para cada ambiente, constata-se que continuam ascendentes, não atingindo a assíntota (Figs. 2-4). Essa situação é clássica em amostragens da fauna de formigas nas regiões tropicais, uma vez que, para grupos hiperdiversos como as formigas, é sempre necessário um grande esforço amostral para que a assíntota da curva seja atingida (SILVA \& SILVESTRE, 2000).

A riqueza estimada para os diferentes sítios de coleta da mata, em cinco dos nove estudados, estão muito acima da riqueza observada (Tab. II). Na capoeira e no reflorestamento este índice mostra menor amplitude diferencial e também menor quando comparado com o fragmento florestal. Este resultado indica a importância de determinar metodologia de coleta que cubra as diferentes condições da área em estudo para melhor detecção da diversidade. A semelhança da riqueza e diversidade entre as três áreas confirma a necessidade de realizar um maior esforço amostral em ambiente com heterogenia topográfica e de vegetação, deixando mais concisas as comparações entre ambientes.

As guildas de formigas encontradas, na mata primária, capoeira e área de reflorestamento, foram: espécies onívoras, predadoras especialistas, predadoras generalistas de serapilheira, formigas legionárias, arborícolas dominantes, dominantes de solo e cultivadoras de fungo (Tab. III). A maioria das formigas

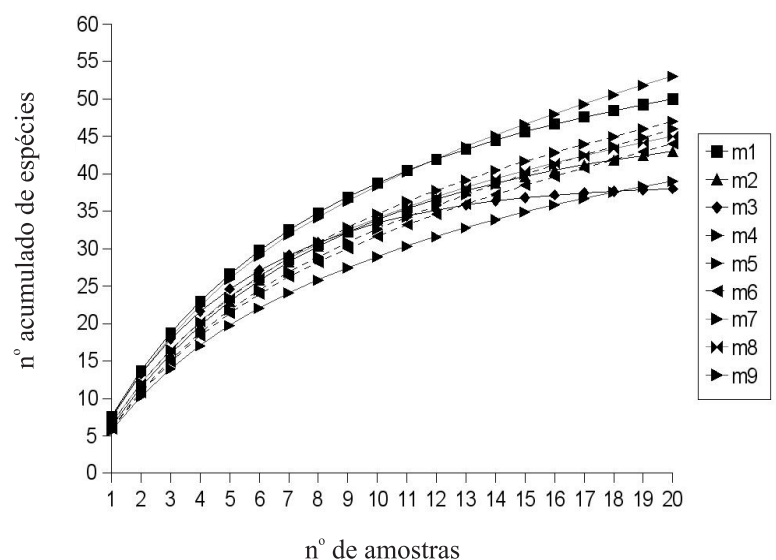

Figura 2. Curvas de riqueza acumulada calculadas para cada série de transectos $(\mathrm{m})$ para formigas capturadas em mata primária, Parque Estadual Mata dos Godoy, Londrina, PR, entre dezembro de 2004 e março de 2005

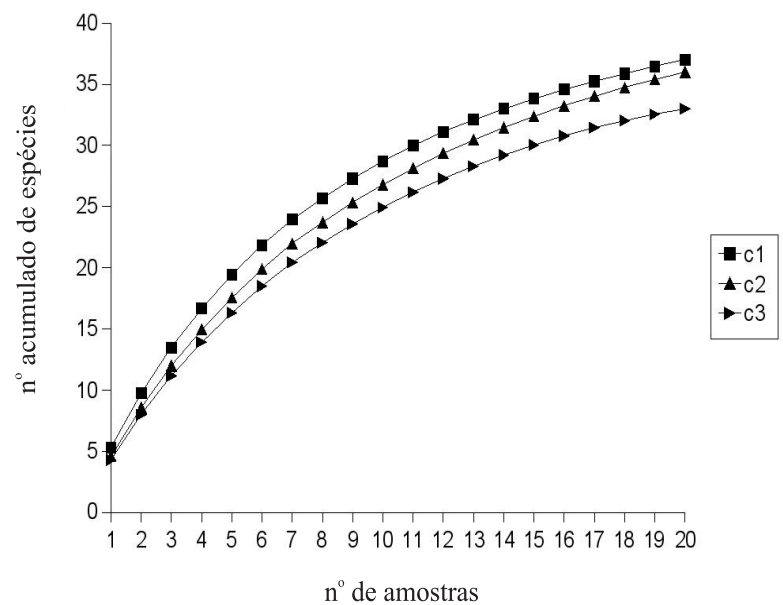

Figura 3. Curvas de riqueza acumulada calculadas para cada série de transectos (c), para formigas capturadas em capoeira, Parque Estadual Mata dos Godoy, Londrina, PR, entre dezembro de 2004 e março de 2005

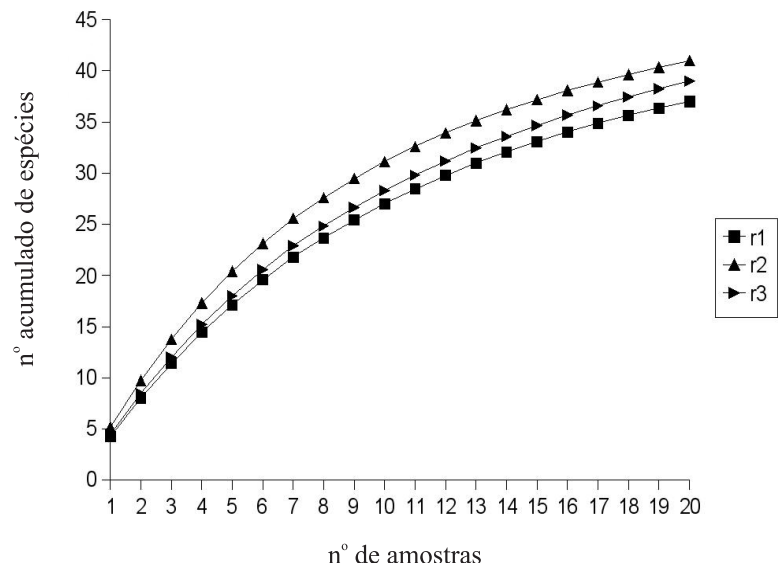

Figura 4. Curvas de riqueza acumulada calculadas para cada série de transectos (r), para formigas capturadas em área de reflorestamento, Parque Estadual Mata dos Godoy, Londrina, PR, entre dezembro de 2004 e março de 2005.

Tabela II. Riqueza observada (Riq. obs) e estimada (Riq. est., Chao 2) de espécies para cada área de coleta dos três ambientes no Parque Estadual Mata dos Godoy, Londrina, PR, entre dezembro de 2004 e março de 2005 (M, mata; C, capoeira; R, reflorestamento).

\begin{tabular}{|c|c|c|c|c|c|c|c|c|c|c|c|c|c|c|c|}
\hline Amostras & M1 & M2 & M3 & M4 & M5 & M6 & M7 & M8 & M9 & $\mathrm{C} 1$ & $\mathrm{C} 2$ & C3 & R1 & R2 & R3 \\
\hline Riq. obs. & 50 & 43 & 38 & 47 & 46 & 44 & 53 & 45 & 39 & 37 & 36 & 33 & 37 & 41 & 39 \\
\hline Riq. est. & 59 & 48 & 38 & 83 & 86 & 77 & 85 & 63 & 46 & 44 & 41 & 35 & 42 & 48 & 48 \\
\hline
\end{tabular}


Tabela III. Classificação dos gêneros de formigas do Parque Estadual Mata dos Godoy, Londrina, Paraná, por guilda, adaptado de DeLABIE et al. (2000) (Arbor, arborícolas dominantes; Cult fungo, cultivadoras de fungo; Dom solo, dominantes de solo; Leg, legionárias; On, onívoras; Pred esp, predadoras especialistas; Pred gen, predadoras generalistas).

\begin{tabular}{lllllll}
\hline Arbor dom & Cult fungo & Dom solo & Leg & On & Pred esp & Pred gen \\
\hline Camponotus & Acromyrmex & Brachymyrmex & Labidus & Brachymyrmex & Discothyrea & Ectatomma \\
Cephalotes & Apterostigma & Camponotus & Nomamyrmex & Camponotus & Eurhopalothrix & Gnamptogenys \\
Crematogaster & Cyphomyrmex & Ectatomma & & Hylomyrma & Leptogenys & Heteroponera \\
Monomorium & Mycocepurus & Linepithema & & Megalomyrmex & Octostruma & Hypoponera \\
Myrmelachista & Myrmicocrypta & Odontomachus & Monomorium & Oxyepoecus & Odontomachus \\
Nesomyrmex & Trachymyrmex & Pachycondyla & Pheidole & Pyramica & Pachycondyla \\
Procryptocerus & & Paratrechina & Rogeria & Strumigenys & \\
Pseudomyrmex & & Pheidole & & Solenopsis & & \\
& & Solenopsis & & & \\
& & Wasmannia & & & \\
\hline
\end{tabular}

encontradas neste estudo nidificam no solo, entretanto, espécies que nidificam em árvores foram coletadas forrageando no solo, mostrando a capacidade desses organismos de explorarem fontes de alimentos mesmo em locais onde a competição (com a mirmecofauna epigéica, por exemplo) pode ser alta. A ocorrência de várias guildas pode indicar a riqueza de nichos ecológicos em fragmento florestal como o do Parque Estadual Mata dos Godoy, o que contribui para o sucesso adaptativo e a preservação de espécies sensíveis às modificações ambientais.

Este estudo pode ser considerado um passo inicial para o conhecimento da mirmecofauna do norte do estado do Paraná. Os resultados obtidos revelam que a fauna de formigas do Parque Estadual Mata dos Godoy é estruturalmente parecida com a de outros ecossistemas de Mata Atlântica do Brasil.

Agradecimentos. A Edson Mendes Francisco, pelo auxílio nas coletas. À CAPES pelo auxílio financeiro que destina ao programa de mestrado da UEL. Ao CNPq pela concessão da bolsa de pesquisador a Jacques H. C. Delabie.

\section{REFERÊNCIAS BIBLIOGRÁFICAS}

ANDERSEN, A. N. 1984. Community organization of ants in the Victorian Mallee. Victorian Naturalist 101:248-251.

1997. Using ants as bioindicators: multiscale issues in ant community ecology. Conservation Ecology 1(1):8.

Belshaw, R. \& Bolton, B. 1993. The effect of forest disturbance on the leaf litter ant fauna in Ghana. Biological Conservation 2:656-666.

Bestelmeyer, B. T.; Agosti, D.; Alonso, L. E.; Brandão, C. R. F.; Brown JR, W. L.; Delabie, J. H. C. \& Silvestre, R. 2000. Field techniques for the study of ground-living ants: an overview, description, and evaluation. In: Agosti, D.; Majer, J. D.; Alonso, L. E. \& Schultz, T. eds. Ants: standart methods for measuring and monitoring biodiversity. Washington, Smithsonian Institution. p.122-144.

Bolton, B. 1995. A new general catalogue of the ants of the World. Cambridge, Massachusetts, Harvard University. 504p. 2003. Synopsis and classification of Formicidae. Memoirs of the American Entomological Institute 71(1):1-370.

Bolton, B.; Alpert, G.; Ward, P. S. \& NASkRecki, P. 2006. Bolton's catalogue of the ants of the world: 1758-2005. Cambridge, Massachusetts, Harvard University. CR-ROM.

Bruhl, C. A.; Mohamed, M. \& Linsenmair, K. 1998. Altitudinal distribution of leaf litter ants along a transect in primary forests in Mount Kinabalu, Sabah, Malaysia. Journal of Tropical Ecology 15:265-277.

Bustos, J. \& Ulloa-Chacón, P. 1997. Mirmecofauna y perturbación en un bosque de niebla neotropical (Reserva Natural Hato
Viejo, Valle del Cauca, Colombia). Revista de Biología Tropical 44(145):259-266.

Colwell, R. K. 2005. EstimateS: Statistical estimation of species richness and shared species from samples. Version 6.01. User's Guide and application. Disponível em: 〈http://viceroy.ebb.uconn.edu/estimates>. Acesso em: 10.2005 .

Delabie, J. H. C.; Agosti, D. \& Nascimento, I. C. 2000. Litter ant communities of the Brazilian Atlantic rain forest region. In: Agosti, D.; Majer, J. D.; Alonso, L. E. \& Schultz, T. R. eds. Sampling ground-dwelling ants: case studies from de world's rain forests. Perth, Curtin University School of Environmental Biology. p.1-17.

Delabie, J. H. C. \& Fowler, H. G. 1995. Soil and litter cryptic ant assemblages of Bahian cocoa plantations. Pedobiologia 39:423-433

Delabie, J. H. C.; Paim, V. R. L. M.; Nascimento, I. C.; Campiolo, S. \& Mariano, C. S. F. 2006. As formigas como indicadores biológicos do impacto humano em manguezais da costa sudeste da Bahia. Neotropical Entomology 35:602-615.

Fernandez, P. 2003. ¿Se mantiene la diversidad de hormigas con el cambio de bosque mesófilo a cafetales? Revista Protección Vegetal 12(2): 17-30.

Fowlen, H. G. 1993. Relative representation of Pheidole (Hymenoptera: Formicidae) in local ground ant assemblages of the Americas. Anales de Biología 9:29-37.

Hölldobler, B. \& Wilson, E. O. 1990. The ants. Cambridge, Belknap/Harvard University. 732p.

Lassau, S. A. \& Hochuli, D. F. 2004. Effects of habitat complexity on ant assemblages. Ecography 27:157-164.

Majer, J. D. \& Camer-Pesci, P. 1991. Ant species in tropical Australian tree crops and native ecosystems - is there a mosaic? Biotropica 23:173-181.

Majer, J. D. \& Delabie, J. H. C. 1994. Comparison of the ant communities of annually inundated and terra firme forests at Trombetas in the Brazilian Amazon. Insectes Sociaux 41: 343-359.

Majer, J. D. \& Коск, A. E. 1992. Ant recolonization of sand mines near Richards Bay, South Africa: an evaluation of progress with rehabilitation. South African Journal of Science 88:31-36.

Marsh, A. C. 1986. Ant species richness along a climate gradient in the Namib Desert. Journal of Arid Environments 11:235-241.

Matos, J. A.; Yamanaka, C. N.; Castellani, T. T. \& Lopes, B. C. 1994. Comparação da fauna de formigas de solo em áreas de plantio de Pinus elliottii, com diferentes graus de complexidade estrutural (Florianópolis, SC). Biotemas 7:57-64.

Noss, R. F. 1990. Indicators for monitoring biodiversity: a hierarchical approach. Conservation Biology 4(4):355-364.

Oliveira, M. A.; Della Lucia, T. M. C.; Araúso, N. S. \& Cruz, A. P. 1995. A fauna de formigas em povoamentos de eucalipto e mata nativa no Estado do Amapá. Acta Amazonica 25(1):11-126.

Oliveira, P. S. \& Brandão, C. R. F. 1991. The ant community associated with extrafloral nectaries in Brazilian cerrados. In: Cutler, D. F. \& Huxley, C. R. eds. Ant-Plant Interactions Oxford, Oxford University. p.198-212. 
Perfecto, I. \& Vandermeer, J. H. 1996. Microclimatic changes and the indirect loss of ant diversity in a tropical agroecosystem. Oecologia 108:577-582

Punttila, P.; Haila, Y.; Pajunen, T. \& Tukia, H. 1991. Colonization of clear-cut forests by ants in the southern Finnish taiga: a quantitative survey. Oikos 61:250-262.

Ramos-Suárez, M. P.; Morales, H.; Ruiz-Montoya, L. L.; SotoPinto, L. \& Rojas-Fernandes, P. 2002. ¿Se mantiene la diversidad de hormigas con el cambio de bosque mesófilo a cafetales? Revista Protección Vegetal 12(2):17-30. (Actas del Simposio Café Y Biodiversidad).

Roth, D. S.; Perfecto, I. \& Rathcke, B. 1994. The effects of management systems on ground foraging ant diversity in Costa Rica. Ecological Applications 4(3):423-436.

Silva, R. R. \& Lopes, B. C. 1997. Ants (Hymenoptera: Formicidae) from Atlantic rainforest at Santa Catarina Island, Brazil: two years of sampling. Revista de Biología Tropical 45:1641-1648.
Silva, R. R. \& Silvestre, R. 2000. Diversidade de formigas (Hymenoptera: Formicidae) em Seara, oeste de Santa Catarina Biotemas 13(2):85-105.

2004. Riqueza da fauna de formigas (Hymenoptera: Formicidae) que habitam as camadas superficiais do solo em Seara, Santa Catarina. Papéis Avulsos de Zoologia 44(1): $1-11$

Torres, J. A. 1984. Diversity and distribution of ant communities in Puerto Rico. Biotropica 16:296-303.

WiLson, E. O. 1976. Which are the most prevalent ant genera? Studia Entomologica 19:187-200.

2003. Pheidole in the New World, a dominant, hyperdiverse ant genus. Cambridge, Massachusetts, Harvard University. 794p.

Wisdom, W. \& Whitford, W. G. 1981. Effects of vegetation change on ant communities of arid rangelands. Environmental Entomology 10:893-897. 